\title{
Die distale Radiusfraktur beim alten Menschen
}

\author{
Thorsten Uzdil, Dara Orangi, Karl Heinrich Winker
}

\section{Zusammenfassung}

Die Traumatologie wird sich bei der verändernden Bevölkerungsstruktur zunehmend mit der Behandlungsstrategie der „distalen Radiusfraktur“ des alten Menschen beschäftigen müssen. Hochgradige Osteoporose, Hautatrophien, akute und chronische Nebenerkrankungen sowie die häufig eingeschränkte Compliance stellen eine große Herausforderung dar. Die Versorgung instabiler distaler Radiusfrakturen mit minimalinvasiven Verfahren, wie der K-Draht-Osteosynthese oder dem Fixateur externe bzw. eine Kombination aus beiden, führt aufgrund der reduzierten Knochenqualität zur Sekundärdislokation oder Implantatlockerungen mit eventueller Infektion und schließlich nach langen Ruhigstellungszeiten zur Heilung in Fehlstellung, zu funktionellen Einschränkungen oder Inaktivitäts- bzw. Reflexdystrophie. Mit dem Einsatz

\section{Einleitung}

Die distale Radiusfraktur ist mit einer Inzidenz von 2/1000 Einwohnern pro Jahr und deren Steigerung auf das 3-4fache bis zum 85.Lebensjahr eine der häufigsten Verletzungen des menschlichen Skelettes überhaupt [1]. Sie entsteht in $90 \%$ der Fälle durch einen Sturz auf die überstreckte Hand. Der Altersgipfel liegt bei Frauen zwischen dem 60. und 80. Lebensjahr und bei Männern zwischen dem 30. und 50.Lebensjahr. Frauen sind dabei im Durchschnitt 4-mal häufiger betroffen als Männer [6], wobei das Lebenszeitrisiko von Frauen bei $21 \%$ und von Männern bei $5 \%$ liegt [1]. Somit ist bei dieser Alters- und Geschlechtsverteilung fast im-

OP-JOURNAL 2005; 21: 186-191

(c) Georg Thieme Verlag KG Stuttgart · New York

formgerechter winkelstabiler Implantate ist die frühzeitige ergo- und physiotherapeutische Behandlung möglich geworden. Trotz verminderter Knochendichte ist die übungsstabile Retention nach offener Reposition und winkelstabiler Plattenosteosynthese distaler Radiusfrakturen und somit die anatomiegerechte Bruchheilung, in nahezu allen Fällen erreichbar. Der streng subchondralen Platzierung winkelstabiler Elemente und der schonenden Reposition über das Implantat selbst kommt dabei eine besondere Bedeutung zu. Mit frühzeitigen Bewegungsübungen und dem schmerzfreien Einsatz der Hand im täglichen Leben wird das Aktivitätsniveau vor dem Unfallereignis wiederhergestellt und der alte Mensch vor der sozialen Abhängigkeit geschützt. Durch Reflexdystrophie hervorgerufene komplizierte Verläufe sind äußerst selten geworden. ble Nachsorge bedürfen festgelegter Versorgungsstrategien:

\section{Diagnostik und Therapie}

Nach einer Nativ-Röntgendiagnostik sollte der Bruch obligat klassifiziert werden. Computertomographische Untersuchungen sind zur Beantwortung offener Fragen beim alten Menschen seltener, zur Beurteilung der intraartikulären Bruchkomponente immer wieder notwendig. Hier haben sich die AO-Klassifikation (Abb.1) wie die Melone-Klassifikation (Abb.2) auch im Hinblick auf die anstehende Therapiewahl bestens etabliert. Anschließend erfolgt die Differenzierung in stabile oder instabile Frakturen nach den Instabilitätskriterien von Jupiter [2]. Für diese Entscheidungsfindung hat sich auch der Aushang im Mädchenfänger unter Durchleuchtungsbedingungen bewährt (Abb.4). Neben den offenen Brüchen und Frakturen mit neurologischen Defiziten werden alle instabilen und dislozierten Frakturen operativ versorgt. Kontraindikationen aus anästhesiologischer Sicht gibt es bei den heutigen Möglichkeiten der Narkoseführung und Regionalanästhesie kaum noch. Die semioperative Therapie mit K-Drähten sollte bei alten Menschen wegen dem erhöhten Risiko von Korrekturverlust durch Implantatlockerung und der Notwendigkeit von langen Ruhigstellungszeiten keine Anwendung mehr finden.

ben erschweren eine optimale Versorgung. Angesichts der demografischen Entwicklung in Deutschland mit den sinkenden Geburtenraten und der verlängerter Lebenszeit stellt eine Behandlung dieser Frakturen aus alterstraumatologischer Sicht eine große Herausforderung dar. Die schnelle Rückkehr zum präoperativen Aktivitätsniveau und die Vermeidung von sozialer Isolation sowie langanhaltender Pflegebedürftigkeit ist das erklärte Ziel und muss über frühzeitige Übungs- und Belastungsstabilität unter Vermeidung von zirkulären Gipsverbänden geschehen. Sowohl diese sozioökonomischen Aspekte als auch das operativ technische Problem und eine komforta-
Instabilitätskriterien nach Jupiter (2)

(zwei Kriterien müssen erfüllt sein)

- dorsale Trümmerzone

- Dorsalkippung $>20^{\circ}$

- axiale Einstauchung $>2 \mathrm{~mm}$

- Mehrfragmentfraktur

- assoziierte Ulnafraktur

radioulnare Instabilität

\section{Konservative Behandlung}

Sollte nach den Instabilitätskriterien von Jupiter ein stabiler körperferner Speichenbruch vorliegen, kommt eine konservative Behandlung in Betracht. Die In- 


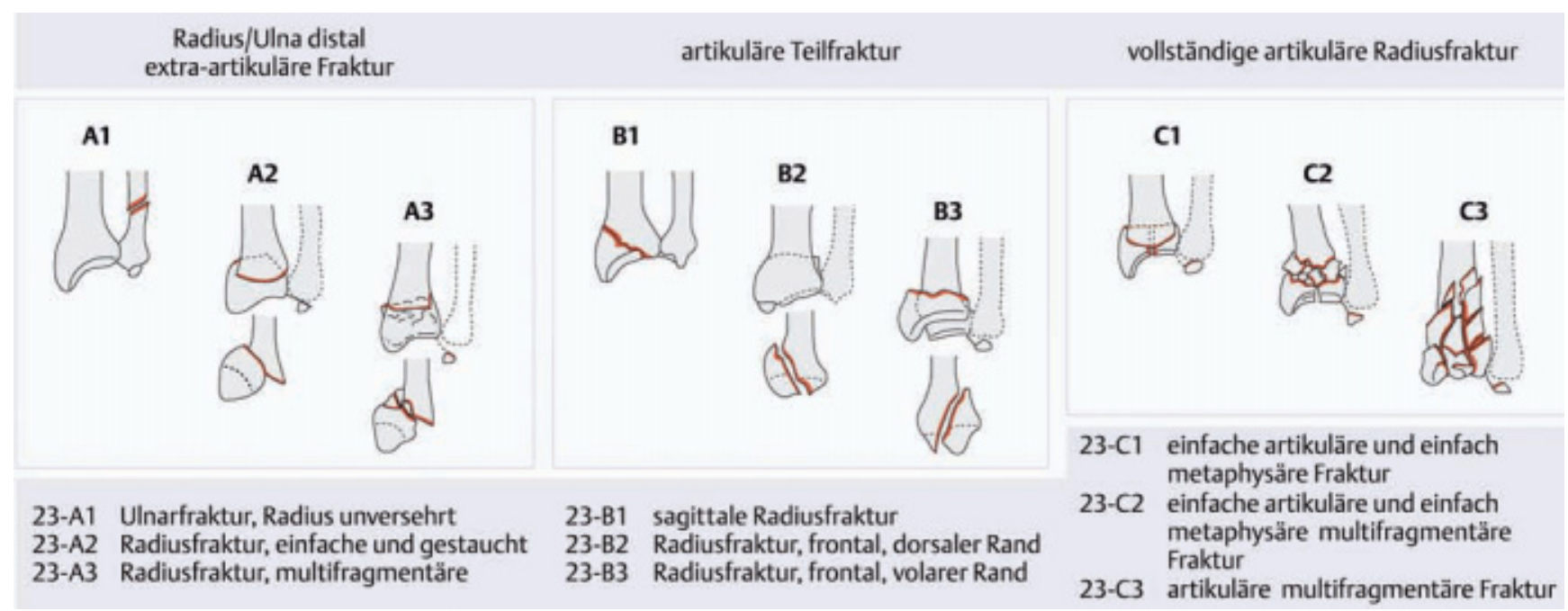

Abb.1 AO-Klassifikation.
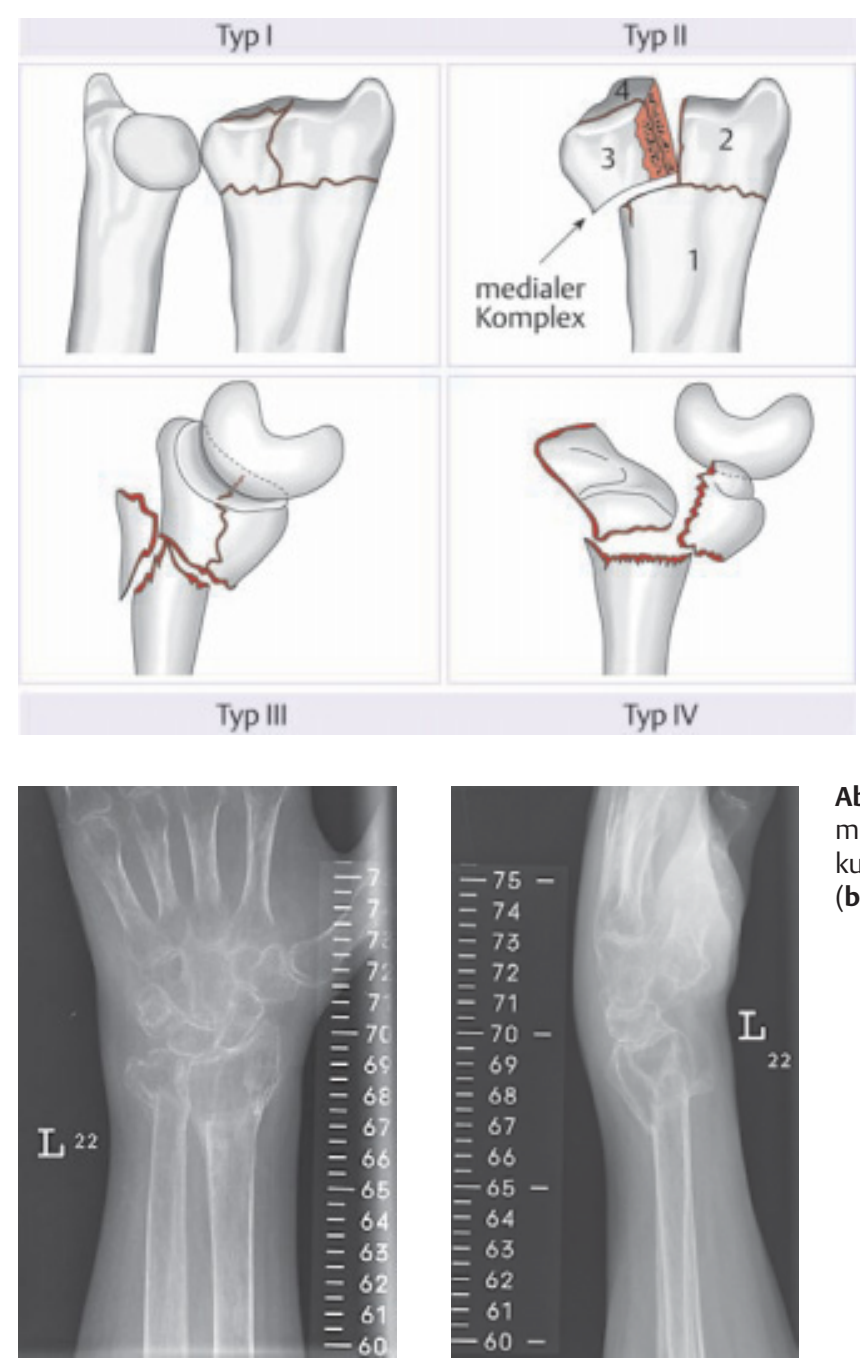

dikation zur klassisch konservativen Therapie bei alten Menschen besteht danach lediglich noch bei nicht dislozierten B1und C1-Frakturen, sowie geringfügig dislozierten A2- sowie allen anderen unverschobenen Frakturen. Diese Frakturtypen
Abb. 3 Reflexdystrophie mit typischem Entkalkungsmuster (a) a-p (b) seitlich.

Abb. 2 Melone-Klassifikation (aus 7).

sind im geriatrischen Patientengut die Ausnahme. Sollte diese Bruchform jedoch bestehen und darüber hinaus die Weichteile eine Gipsbehandlung zulassen, erfolgt die Gipsanlage im Mädchenfängeraushang ohne Gewicht und ohne Repo- sitionsmanöver. Die Beweglichkeit der Grundgelenke der Langfinger und des Daumens muss für die frühzeitige Benutzung gewährleistet bleiben (Abb.4). In die Bewegungsanleitung sollte auch das Ellenbogen- und Schultergelenk mit einbezogen werden. Der zirkuläre Unterarmgips wird dann am Anlagetag ulnar gespalten und für 4 Wochen getragen. Zur Beurteilung der Weichteilverhältnisse kann eine Gipsabnahme vor der Röntgenkontrolle am 7.Tag stattfinden. Eine überbetonte Flexionsstellung des Handgelenkes im Gips sollte wegen der Gefahren eines Kompressionssyndroms des N. medianus vermieden werden. Nach Abnahme des Gipses beginnt eine intensive Ergo- und Physiotherapie zur schnellen Wiederherstellung des Ausgangsaktivitätsniveaus und Reflexdystrophieprophylaxe. Voraussetzung für eine komplikationslose, erfolgreiche konservative Behandlung sind die richtige Auswahl und Unterscheidung zwischen stabilen und instabilen Frakturen und der Ausschluss von allgemeinen Kontraindikationen. An dieser Stelle werden höhergradige periphere arterielle Verschlusskrankheiten, Polyneuropathien, Hautläsionen bei Pergamenthaut, Paresen der betroffenen oder kontralateralen oberen Extremität und Thrombophilien genannt. Röntgenkontrollen werden am 7.Tag sowie nach Ausheilung empfohlen. Man sollte sich nicht scheuen, Patienten mit hochgradiger Altersdemenz ohne wesentliche Schmerzäußerung auch sofort funktionell ohne Ruhigstellung $\mathrm{zu}$ betreuen, um damit mögliche Komplikationen durch die Immobilisierung zu verhindern.

Mögliche Hilfeleistungen zum Erhalt des labilen körperlichen und seelischen $\mathrm{Zu}$ - 


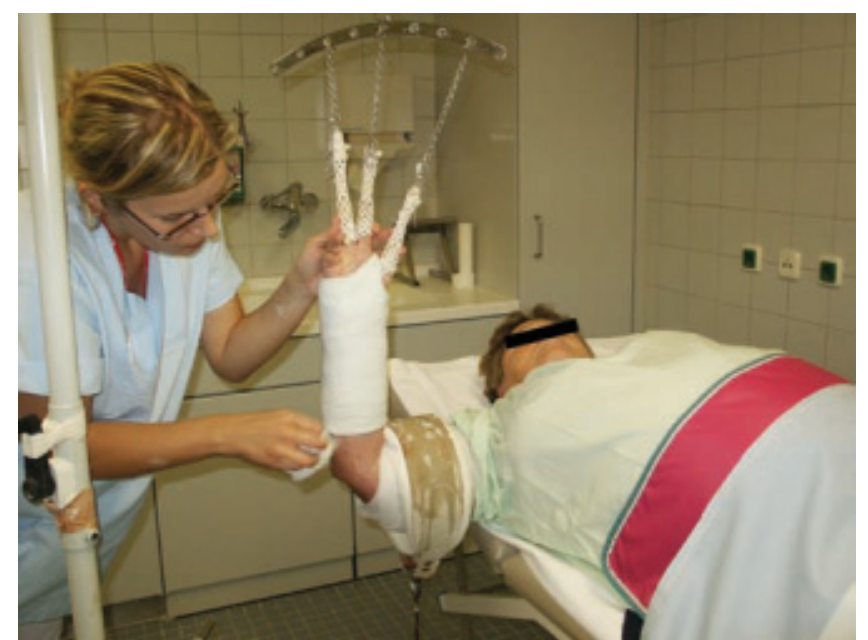

Abb.4 Aushang im Mädchenfänger und Gipsanlage.

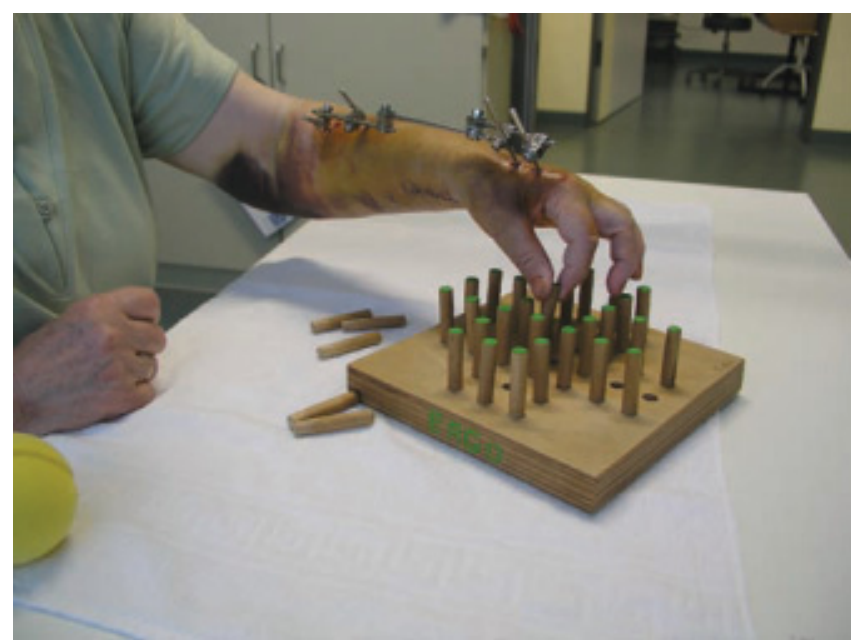

Abb.5 Ergotherapie mit Fixateur externe. standes werden durch Sozialdienste der Krankenhäuser und ambulante Pflegedienste ausgeschöpft. Nicht zuletzt sollte jedoch für einen Erfolg dieser Behandlung die Familie frühzeitig in die Problematik eingeweiht und aktiv eingebunden werden.

\section{Operative Behandlung}

Hauptziele der operativen Behandlung des alten Menschen sind die gewebeschonende Reposition mit achsen- und gelenkgerechtem Ausgleich der radialen Gelenkfläche und die frühfunktionelle gipsfreie Beübung (Abb.5). Somit kann der Wundheilungsstörung, der Reflexdystrophie (Abb.3) und schlechten funktionellen Ergebnissen vorgebeugt werden. Als Verfahren stehen die winkelstabile dorsale und palmare Plattenosteosynthese und die Varianten des Fixateur externe zur Verfügung. Die perkutane Kirschner-Drahtosteosynthese empfiehlt sich beim alten Menschen mit verminderter Knochenqualität nur im Ausnahmefall.

\section{Fixateur externe}

Die primäre Osteosynthese und Ausbehandlung im Fixateur externe stellt hohe Anforderungen an die Knochenqualität, an die Akzeptanz des Betroffenen und an die Pin-Pflege im ambulanten Bereich. Deshalb kann diese Methode nur selten beim alten Menschen erfolgreich realisiert werden. Pin-Infekte und Verfahrenswechsel sowie schlechte röntgenologische Ergebnisse werden nicht selten beobachtet. Der Vorteil dieses Verfahrens liegt in der Möglichkeit einer gewebeschonenden Reposition unter Ausnutzung der Ligamentotaxis und der Vermei- dung von Gipsverbänden bei den häufig schwierigen Hautverhältnissen. Die Ergotherapie der Hand gelingt bei liegendem Fixateur externe sehr gut (Abb.5).

\section{Dorsale Platte}

Als Abstützplattenosteosynthese hat sich dieses Verfahren bei den instabilen Extensionsfrakturen mit dorsaler Trümmerzone und Gelenkbeteiligung bewährt. Hier sind über den dorsalen Zugang Gelenkrekonstruktionen und die Schaffung von übungsstabilen Verhältnissen möglich [5]. Als Nachteil wird der technisch anspruchsvolle Zugang und die in der Regel notwendige Materialentfernung wegen der Strecksehnenirritation empfunden. Dieses Problem der Implantatdeckung wird bei den alten Menschen mit Haut- und Muskelatrophie noch verstärkt. Hier wird eine weitere Optimierung von Plattendesign und -dimension möglicherweise Auswege schaffen.

\section{Palmare winkelstabile Platte}

Mit winkelstabilen Implantaten ist eine übungsstabile Osteosynthese bei Flexions- aber auch nahezu allen instabilen Extensionsfrakturen möglich geworden. Über den technisch einfachen radiopalmaren Zugang können, neben der anatomischen Gelenkrekonstruktion, dorsale Trümmerzonen durch die Winkelstabilität des Implantates, selbst bei höhergradigen Instabilitäten sicher überbrückt werden $[2,4,7]$. Spongiosaplastiken sind in der Regel selbst bei ausgedehnten Trümmerzonen nicht notwendig [7]. Auch Materialentfernungen werden wegen der guten palmaren Gewebedeckung der Implantate beim alten Menschen nicht durchgeführt. Zahlreiche Veröffent- lichungen konnten die Vorzüge dieses Vorgehens herausarbeiten [4]. Insbesondere überzeugen die guten röntgenologischen und funktionellen Ergebnisse, die kürzeren Behandlungszeiten, geringe Komplikationsraten wie die praktisch nicht mehr nachweisbare Reflexdystrophie und der gestiegene Patientenkomfort [3].

\section{Eigenes Vorgehen [4]}

Trümmerzonen bei höhergradiger Osteoporose, schlechte Weichteilverhältnisse bei Hautatrophie und die zunehmend schlechter werdende Compliance stellen hohe Anforderungen an das operative Therapiemanagement. Hierbei hat sich bei instabilen Extensionsfrakturen des alten Menschen der nachfolgende therapeutische Stufenplan etabliert. Zunächst wird die frische Verletzung ohne Repositionsmanöver im Mädchenfänger mit ca. $5 \mathrm{~kg}$ über 15 Minuten hinweg ausgehängt (Abb.4). Nach Anlage eines zirkulären Unterarmgipses, mit einem Zeitaufwand von ca. 10 Minuten, wird dieser nach der erfolgten OP-Vorbereitung gespalten. Der operative Eingriff wird dann je nach Tageszeit in den nachfolgenden $24 \mathrm{~h}$ möglichst in Regionalanästhesie durchgeführt. Bei vorliegender hochgradiger Osteoporose und Hautatrophie wird vor dem eigentlichen Eingriff ein Fixateur externe angelegt. Hierbei sollte die Anordnung der Pins eine maximale Supination bei gestrecktem Ellenbogen zulassen. Dies hat den Vorteil, dass jegliche Distraktion und Reposition direkt über knöcherne Strukturen erfolgen können. Die problematischen Weichteile bleiben also diesbezüglich unberührt. Bei der anschließenden winkelstabilen Plattenosteosynthese über den radiopalmaren 

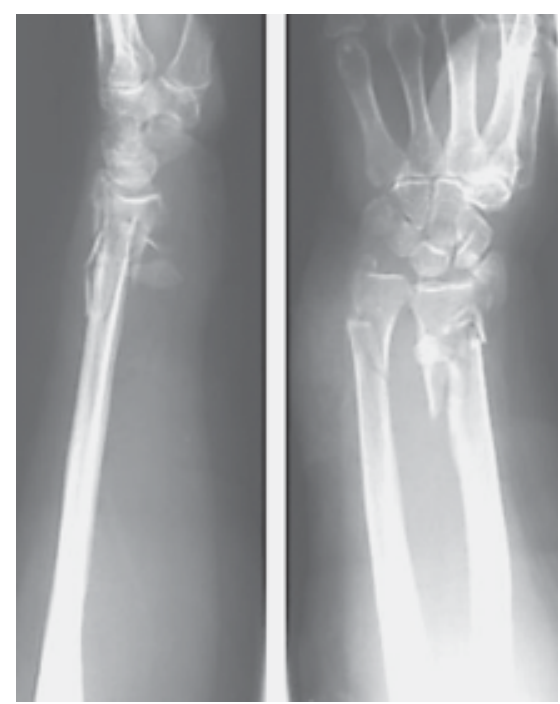

Abb. 6 Distale Unterarmfraktur vom AO-Typ 23-C2/A1 bei Osteoporose.

Zugang wird als wichtigster Schritt der Operation darauf geachtet, dass die winkelstabilen Kraftträger des Plattenquerschenkels unmittelbar in der subchondralen Schicht der radialen Gelenkfläche zu Liegen kommen (Abb.11). Diese Region ist selbst bei hochgradiger Osteoporose als Wiederlager ausreichend geeignet. Bei Gelenkfrakturen kann es hilfreich sein. In radioulnarer Richtung unmittelbar subchondral einen temporären K-Draht zu platzieren (Abb. 7), der die gelenkbildenden distalen Fragmente zu einem Block verbindet und am Ende der Operation wieder entfernt oder durch eine kanülierte Schraube ersetzt wird. Nach erfolgter Plattenosteosynthese in unten genannter Weise wird in Abhängigkeit von den vorliegenden lokalen Weichteilverhältnissen, aber auch der Compliance, entschieden, ob der Fixateur bis zum Fadenzug belassen oder unmittelbar nach der Operation entfernt und durch eine Gipsschiene ersetzt wird. Nach dem Fadenzug sollte der Patient ohne jegliche externe Fixation auskommen.

\section{OP-Schritte (Abb.11 -16)}

OP-Ablauf

1. radiopalmarer Zugang radial der Sehne des M. flexor carpi radialis

2. Positionierung der Platte im a/pStrahlengang monokortikal mit zwei K-Drähten über winkelstabile Bohrhülsen subchondral

3. Positionierung der K-Drähte im seitlichen Strahlengang subchondral dann Vorschub bikortikal

4. Fixierung des Querschenkels durch Schrauben und Austausch der K-Drähte durch winkelstabile Bolzen

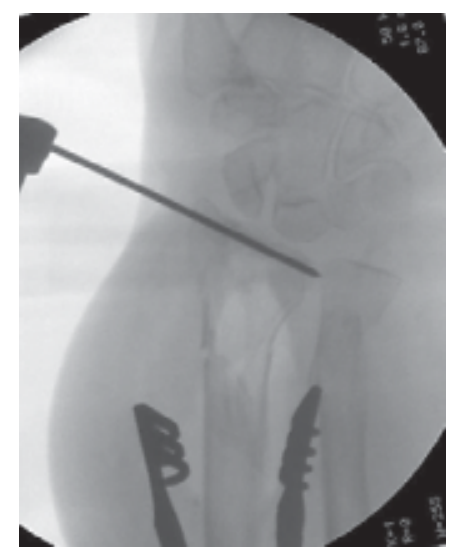

Abb. 7 subchondrale Platzierung eines K-Drahtes in radioulnarer Richtung.

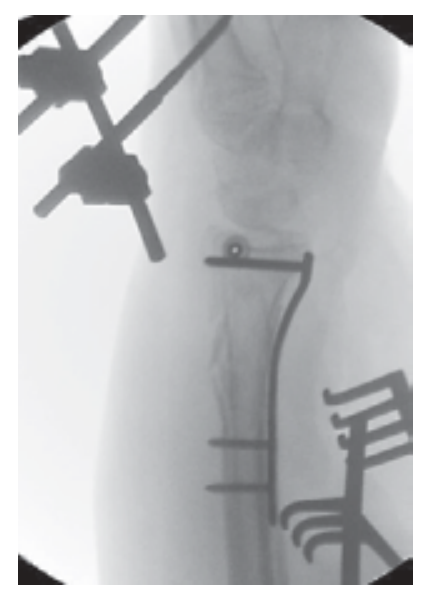

Abb.9 Die winkelstabilen Kraftträger der Platte liegen direkt unter dem K-Draht bzw. der kanülierten Schraube

5. Reposition mit der Platte und Fixation des Längsschenkels zentrisch in einem Langloch

6. Repositionsoptimierung über den Drehpunkt dieser Schraube und Schaffung anatomischer Verhältnisse im DRUG

Die Freilegung der Fraktur erfolgt über den radiopalmaren Zugang mit einem längsverlaufenden Hautschnitt von der distalen Handgelenksbeugefalte entlang der Sehne des M. flexor carpi radialis (FCR) ca. $5 \mathrm{~cm}$ nach proximal. Nach Blutstillung und Darstellung dieser Sehne, wird diese mit einem Sehnenhaken nach ulnar gehalten, die Faszia antebrachii darunter inzidiert und dann der $\mathrm{M}$. pronator quadratus radial der tiefen Beuger stumpf unter Beachtung der A. radialis dargestellt. Erst jetzt wird - wenn überhaupt - die Blutsperre zur Verkürzung der Ischämiezeit geschlossen. Nach Abtrennung des M. pronator quadratus

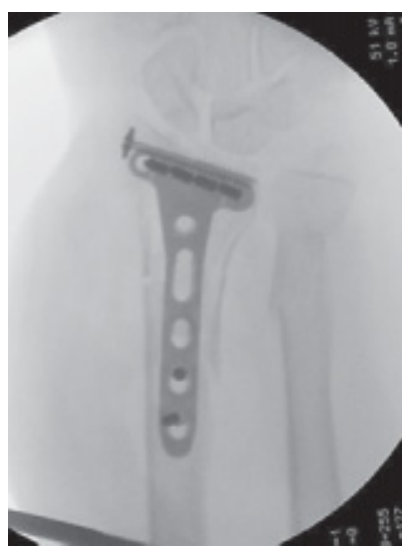

Abb. 8 Nach winkelstabiler Plattenosteosynthese mit der 2,4-mm Radiusplatte wird der KDraht durch eine kanülierte Schraube ersetzt.

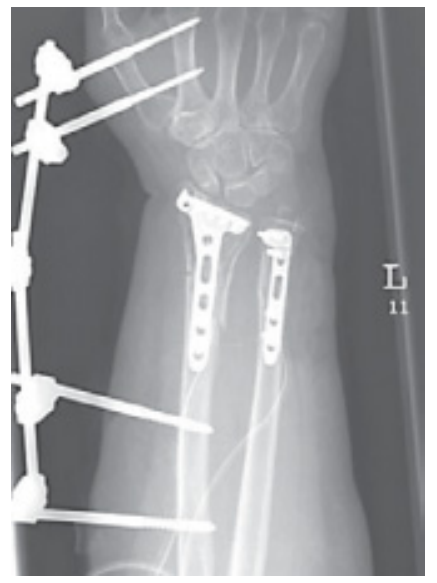

Abb.10 Postop. C2/A1-Fraktur mit winkelstabilen Implantaten 2,4 mm und Fixateur externe.

am radialen Ansatz lässt sich die Fraktur explorieren.

Die Positionierung der Platte erfolgt unter BV-Kontrolle, wobei die Fixierung des Querschenkels über zwei winkelstabile Bolzen mit zwei K-Drähten zunächst monokortikal realisiert wird. Somit sind Positionsänderungen der K-Drähte im seitlichen Strahlengang möglich, ohne dass eine Verschiebung der Platte im a/ p-Strahlengang eintreten kann. Nach Drehung des Unterarmes in den seitlichen Strahlengang werden eventuelle Korrekturen der monokortikal eingebrachten K-Drähte durchgeführt, sodass diese unmittelbar subchondral liegen. Der Vorschub bis zur bikortikalen Verankerung sollte zur optimalen Positionierung unter Durchleuchtung erfolgen.

Die Fixierung des Querschenkels der Platte gelingt am besten über eine Schraube zwischen den winkelstabilen Bolzen im 


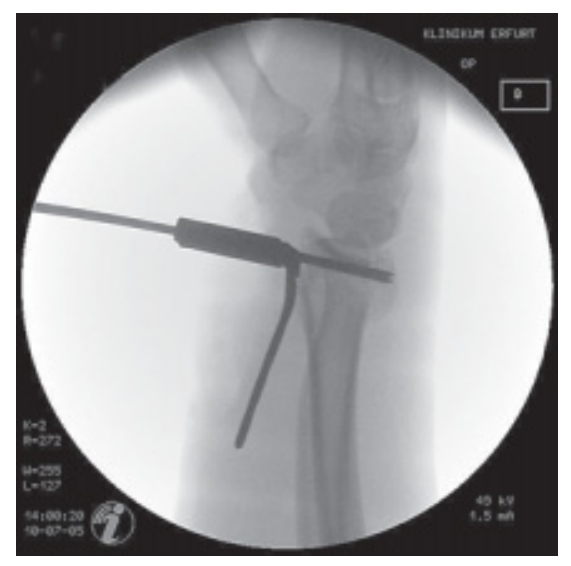

Abb.11 K-Draht subchondral.

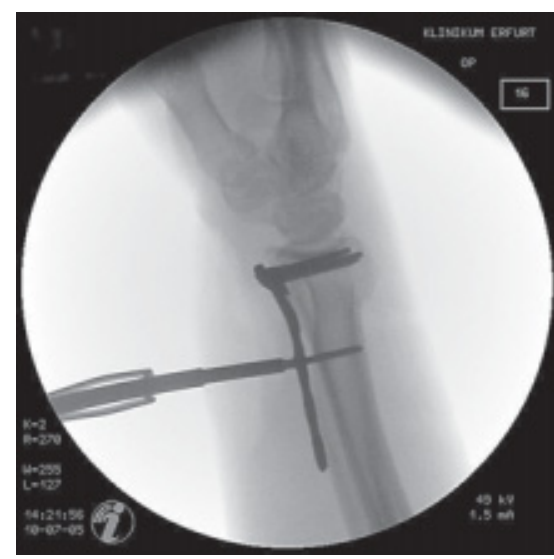

Abb. 14 Reposition der Fraktur mit der Platte durch Fixierung des Längsschenkels.

Sinne einer Zugschraube. Hierbei kann es hilfreich sein, den Querschenkel mit einem Stößel an des distale Fragment zu drücken. Dann erfolgt die ulnare und radiale Fixierung des Querschenkels, wobei das radiale Loch dem Processus stylo-

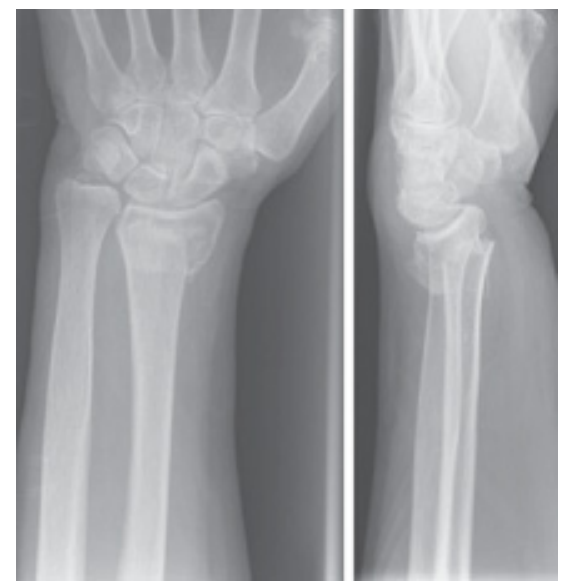

Abb.17 Fall 1: distale Radiusextensionsfraktur AO-Typ A3.

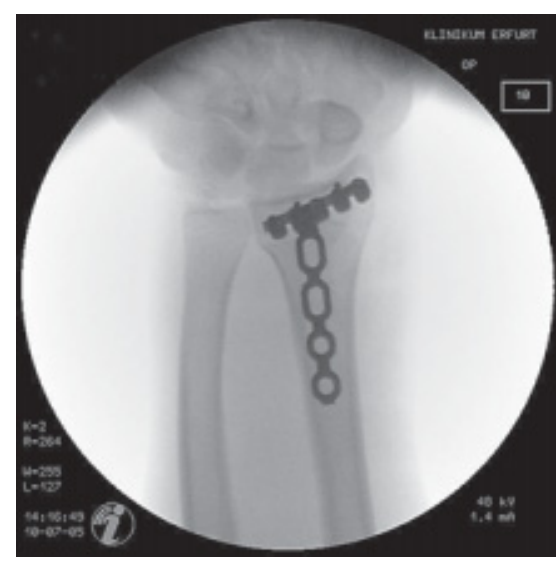

Abb.12 Schraubenfixierung des Querschenkels und Austausch der K-Drähte durch winkelstabile Elemente.

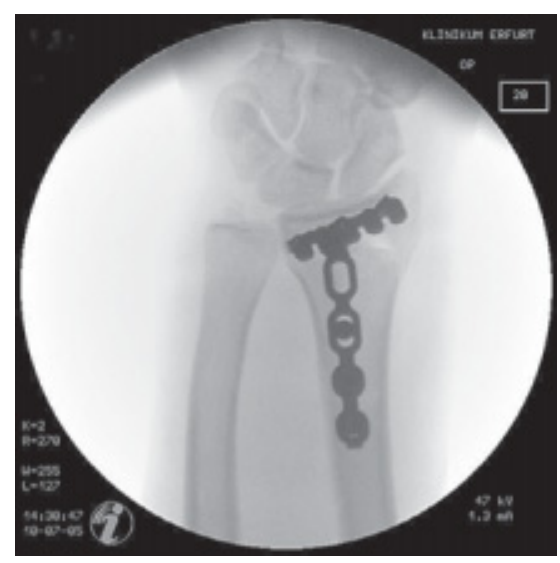

Abb.15 Achsen- und gelenkgerechte Verhältnisse einer A3-Fraktur im a/p-Strahlengang.

ideus radii anliegen muss. Dies erreicht man über einen Biegebolzen, wobei an dieser Stelle die A. radialis nicht interponiert werden darf. Die winkelstabilen Bolzen bzw. Schrauben (je nach Implantat) sollten beim Austausch der K-Drähte

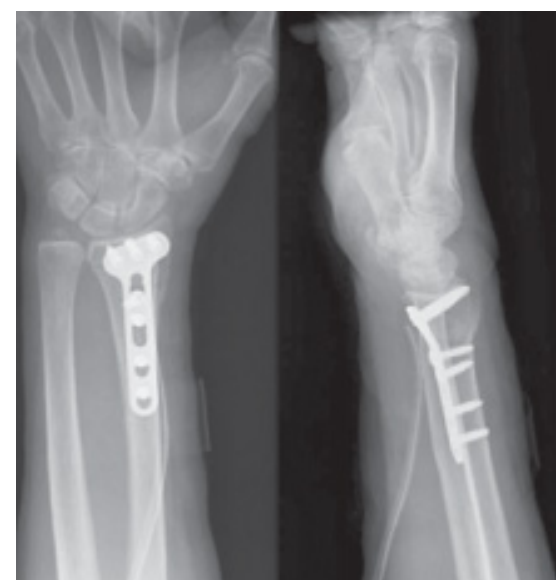

Abb.18 Fall 1: Versorgung mit der winkelstabilen $3,5 \mathrm{~mm}$ LCP.

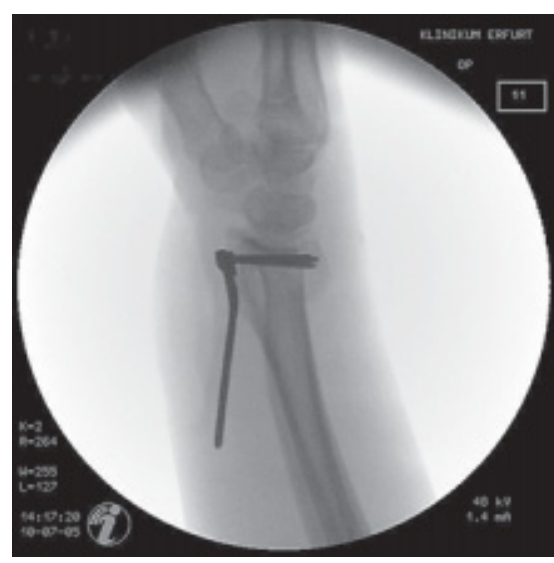

Abb.13 Die Kraftträger des Plattenquerschenkels dürfen die dorsale Kortikalis nicht überschreiten.

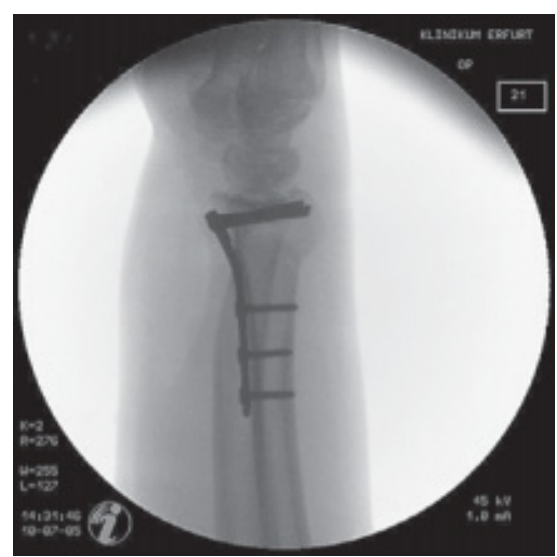

Abb.16 Durch das winkelstabile Implantat ist eine frühfunktionelle Beübung möglich.

so gewählt werden, dass sie an der dorsalen Kortikalis nicht überstehen (Abb.13).

Die eigentliche Reposition erfolgt nun weichteilschonend nur durch Andrücken des Längsschenkels der Platte an den Ra-

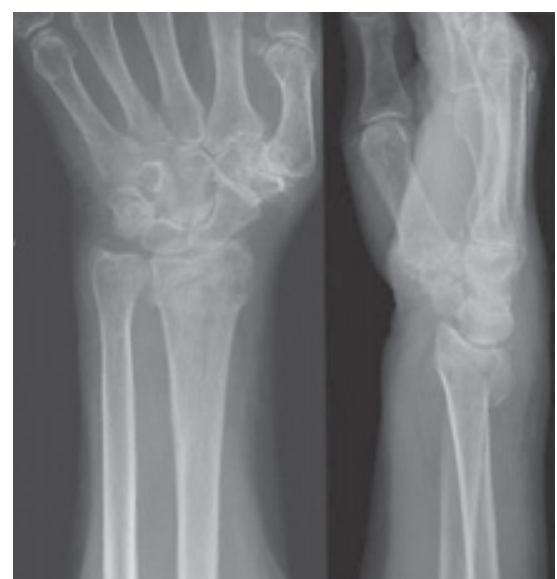

Abb.19 Fall 2: distale Radiusextensionsfraktur Typ 23-A3. 


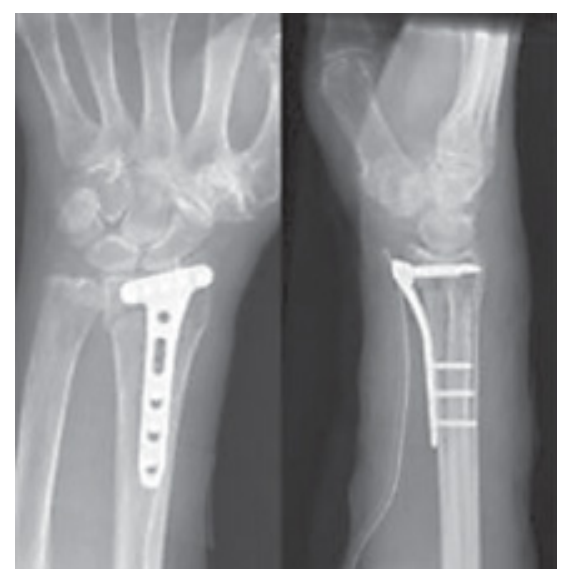

Abb. 20 Versorgung mit der winkelstabilen 2,4 mm distalen Radiusplatte.

\section{Mögliche operationstechnische Fehler}

Dorsal überstehende Schrauben bzw. Bolzen am Querschenkel der Platte führen nicht selten zur Irritation der Strecksehnen und machen eine Materialentfernung notwendig. Deshalb sollten diese Kraftträger die dorsale Kortikalis nicht überschreiten. Irritationen der APL- und EPL-Sehnen werden durch abstehende Plattenquerschenkel am Proc.styl.radii verursacht, welche durch das Anbiegen des radialen Plattenloches vermieden werden können. Auch Irritationen des distalen Radioulnargelenkes werden durch zu ulnare Platten- und Schraubenlage des Querschenkels beobachtet. Sehr selten kommt es zu einer intraartikulären kung der beschrieben Komplikationen. So gehört heute die vor allem palmare winkelstabile Plattenosteosynthese zum festen Therapieregime und ist in vielen großen unfallchirurgischen Abteilungen zum Ausbildungseingriff geworden.

\section{Literatur}

1 Ismail AA, Pye SR, Cockerill WC. Incidence of limb fracture across Europe: results from the EPOS. Osteoporos Int 2002; 13 (7): 565 - 571

2 Jupiter JB. Complex articular fractures of the distal radius: classification and management. J Am Acad Orthop Surg 03: 119-129

3 Krimmer H, Pessenlehner C, Haßelbacher K, Meier M, Roth F, Meier R. Palmare winkelstabile Plattenosteosynthese der instabilen distalen Radiusfraktur. Der Unfallchirurg 2004; 06: 460 - 467
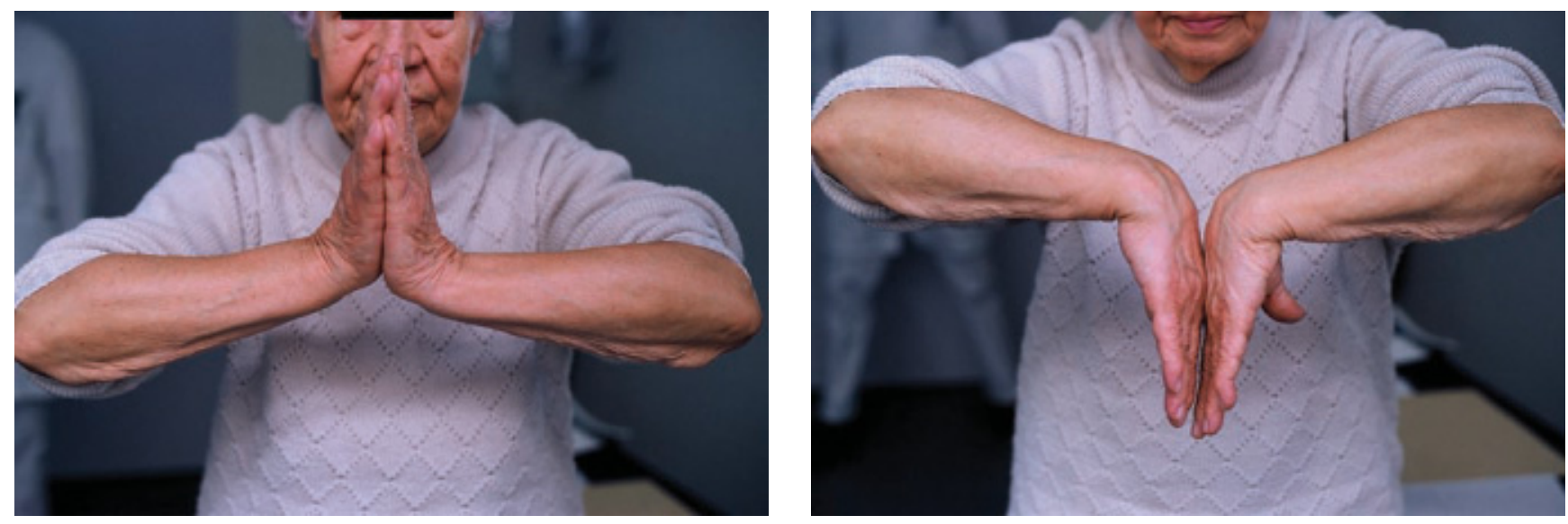

Abb. 21 (a, b): frühfunktionelle Beübung der 89-jährigen Patientin.

dius. Dann wird diese zentrisch mittels Schraube in einem Langloch fixiert, wobei über den Drehpunkt dieser Schraube die Reposition fallweise optimiert werden kann. Insbesondere wird an dieser Stelle darauf geachtet, dass der radioulnare Index bzw. das Längenverhältnis zwischen Radius und Ulna ausgeglichen werden. Die restlichen Schrauben im Längsschenkel sollten die dorsale Kortikalis nur unwesentlich überschreiten.

Nach Öffnung der Blutsperre mit einer Maximalischämiezeit von unter 30 Minuten erfolgt nochmals eine Blutstillung und abschließend eine obligate Durchleuchtungsuntersuchung zur Beurteilung der Osteosynthese und Ausschluss von Begleitverletzungen (Instabilität einer Ulnafraktur; distales Radioulnargelenk; SLDissoziation; Scaphoidfraktur). Dann wird die Nachbehandlung festgelegt. Der Wundverschluss erfolgt durch subkutane Einzelknopf- und kutane Rückstichnaht ohne Verschluss der Faszia antebrachii. Eine routinemäßige Spaltung des Karpaltunnels findet nicht statt.
Implantatlage am Querschenkel des Implantates durch die zu subchondrale Platzierung der Kraftträger, insbesondere bei C-Frakturen. Hier sollte darauf geachtet werden, dass die winkelstabilen Elemente im Querschenkel der Platte sicher außerhalb von Frakturlinien verankert werden.

\section{Schlussfolgerung}

Auch und gerade alte Menschen sind auf die möglichst ungestörte Funktion ihrer Hände besonders angewiesen. Die konservative Behandlung der so häufigen distalen Radiusfraktur beinhaltet eine mindestens 4-wöchige Ruhigstellung mit allen möglichen Folgeerscheinungen wie Bewegungseinschränkung, Hautproblemen, Reflex- oder Inaktivitätsdystrophie, Karpaltunnelsyndrom, Ruptur der EPL-Sehne und natürlich die eingeschränkte Selbstständigkeit. In den letzten 10 Jahren etablierte moderne Operationsmethoden bei den häufigsten beim alten Menschen beobachteten Frakturtypen am distalen Radius (A3, B1-3, C1 und C2) führten zu einer dramatischen Sen-
4 Pabst T, Uzdil T, Winker KH. Die palmare Plattenosteosynthese bei distaler Radiusfraktur: Indikation und Technik. OP-Journal 2003; 19: $42-50$

5 Siebert HR, Klonz A. Distale Radiusfraktur. Der Unfallchirurg 2005; 02: 135-152

6 Wiemer P, Köster G, Felderhoff J, Weber U. Frakturen am distalen Radius, Wandel der therapeutischen Strategien; Orthopädie 1999; 28: 846-852

7 Winker KH. Winkelstabile Plattensysteme am distalen Radius: Lösungsmöglichkeiten und Probleme. Akt Traumatol 2005; 35: $151-154$

\section{Dr. med. med. Thorsten Uzdil} Chirurg

Dr. med. med. Dara Orangi

Oberarzt

Prof. Dr. med. Karl Heinrich Winker Chefarzt

Klinik für Unfallchirurgie Hand- und Wiederherstellungschirurgie

HELIOS Klinikum Erfurt

Nordhäuser Str. 74

D-99089 Erfurt 